\title{
Systemic lupus erythematosus clinically resembling multiple sclerosis and with unusual pathological and ultrastructural features
}

\author{
I NGR I D V.ALLEN, J.H. D. M I L L A R, J . K I R K, \\ A N D ROSEMARY K. A. SHILLINGT O N \\ From the Institute of Neurological Sciences, Royal Victoria Hospital, and Department of Pathology \\ (Neuropathology), Queen's University, Belfast
}

SUMMARY A case of systemic lupus erythematosus is described which clinically resembled multiple sclerosis and in which the lesions were restricted to the central nervous system. The necropsy findings of vascular thickening and necrosis in the spinal cord and in a posterior nerve root explain the main clinical abnormalities. Clinical signs of the terminal peritonitis secondary to cholecystitis were absent or minimised probably because of the steroid therapy and spinal cord necrosis. Primary demyelination was not demonstrated though electronmicroscopy revealed lattice fibrillar inclusions within a few myelin sheaths. An unusual ultrastructural feature was the finding of "rod-shaped tubular bodies" in large numbers in the endothelial cells of cerebral blood vessels. The incidence and morphology of these organelles are compared with those of the intracisternal tubuloreticular structures (TRS) commonly found in systemic lupus erythematosus.

The involvement of the central nervous system in systemic lupus erythematosus (SLE) was emphasised by Osler (1900) in the series of patients which he reported. Neurological complications are recognised as an important cause of death in this disease (Feng et al., 1973) and may develop because of primary involvement of the nervous system, secondary to the disease affecting other organs, or because of the effects of therapy on the nervous system. There are several references in the literature (Fulford et al., 1972; April and Vansonnenberg, 1976) to SLE resembling multiple sclerosis. The reported presence of high levels of viral antibodies (particularly to paramyxoviruses) and of demyelination in both diseases has led to the supposition that there may be a common pathogenetic factor. This view is strengthened by a report of homozygous twins one of whom had SLE, the other multiple sclerosis (Holmes et al., 1967). The present case is reported because of its clinical resemblance to multiple sclerosis, and because of the rarity of reports of central nervous system involvement as the only or the earliest manifestation of SLE. The case provided an

Address for reprint requests: Dr I. V. Allen, Institute of Pathology, Royal Victoria Hospital, Grosvenor Road, Belfast, Northern Ireland. Accepted 14 November 1978 opportunity for a detailed pathological study of the spinal cord and for electronmicroscopy on relatively fresh brain material as necropsy was performed three hours after death.

\section{Case report}

The patient, a 65 year old housewife, was admitted to the Royal Victoria Hospital, Belfast, on 29 July 1975 with severe chest pain. She had a history of ischaemic heart disease, and the pain was considered to be cardiac in origin. A few days after admission she complained of a marked increase in weakness of her legs and inability to pass urine. One year previously she had been diagnosed as suffering from ischaemic heart disease and since then she had noticed that her legs were becoming weaker, with numbness and pain on exertion. She also complained of dyspnoea on exertion and of attacks of nocturnal dyspnoea. There was no history of abdominal pain but she complained of occasional vomiting.

She had suffered from intermittent diplopia for six months 30-35 years previously. In September 1972 she was admitted to hospital complaining of weakness and numbness of the left leg. The numbness spread up to the level of the waist and the 
arms felt heavy. These symptoms lasted for a few weeks. In November 1972, she developed vertigo and deafness in the right ear which recovered over a period of weeks. In July 1973, she lost the vision of the left eye over a period of a few days. This was thought to be caused by occlusion of the central retinal artery. There was no recovery of vision.

On examination there were xanthelasmas around both eyes. The blood pressure was $120 / 75 \mathrm{mmHg}$, pulse rate 112 per min and the jugular venous pressure was not raised. Fine crepitations were heard at both lung bases. The left eye was blind with no perception of light. Its optic disc was pale. Cranial nerves were otherwise normal. There was no abnormality in the arms except for slight generalised weakness. The legs were very weak (left particularly). The left knee jerk and right ankle jerk were absent, and both plantar reflexes were extensor. Sensation to pinprick was absent to a definite level at T4 dermatome. Some pain was felt in the left leg. Proprioception was absent in the left leg but was normal in the right leg. Femoral and popliteal pulses were difficult to feel but posterior tibial and dorsalis pedis pulses were palpable.

Radiography of the chest showed moderate left heart enlargement, no evidence of dissecting aneurysm of aorta, and considerable elevation of the right diaphragm. An ECG on 26 August 1975 was normal; on 1 September it showed ischaemic changes in inferolateral leads. There was abnormality of serum enzymes. A myelogram was normal. The CSF contained 15 lymphocytes/ $\mu 1$, protein $0.6 \mathrm{~g} / 1$, WR negative; Lange curve 0000000 , LE cells were seen in blood on six occasions; ANF was positive; ESR $100 \mathrm{~mm} / \mathrm{hr}$, and bone marrow was hyperplastic in keeping with the level of steroid therapy (see below). The WR was negative; VDRL negative; RPCFT negative; and FTA/ABS negative.

A diagnosis of systemic lupus erythematosus was made with the brunt of the lesions affecting the central nervous system as in previously reported cases (Fulford et al., 1972). The chest pain corresponded to the sensory level and was considered to be neuritic. With steroid treatment there was a slight improvement in power in the legs and, strikingly, the vision in the left upper quadrant of the left eye returned and she could read 6/36 despite the fact that the left eye had been totally blind for two years. The ESR also fell to normal limits. However, she complained of recurrent severe chest pain and, apart from chest infection and the sensory level, no adequate explanation was found. Towards the end of January 1976 the character of the pain changed. She now complained of generalised colicky abdominal pain without nausea or vomiting and without epigastric tenderness. This pain lasted three days. She died on 27 February 1976. For two days before her death she complained of severe central chest pain which radiated down the left arm.

\section{NECROPSY: MATERIAL AND METHODS}

Necropsy was carried out three hours after death. Blocks for histology were fixed in $10 \%$ buffered formalin. Paraffin sections were cut at $7 \mu \mathrm{m}$, frozen sections at $15 \mu \mathrm{m}$. Fixed sections of brain, optic nerves, and spinal cord were stained using the following techniques: haematoxylin and eosin (H and E), Spielmeyer/fat, Woelcke, Weigert's elastic tissue stain. Sections of kidney were stained by $\mathrm{H}$ and $\mathrm{E}$ and Martius' scarlet/blue for fibrin. Blocks for electronmicroscopy (grey and white matter from right and left frontal and temporal lobes) were trimmed to 1-2 mm cubes and immediately fixed in cold $2.5 \%$ glutaraldehyde in $0.1 \mathrm{M}$ s-collidine buffer. After post-fixation in osmium tetroxide the blocks were dehydrated and embedded in Epon 812. Areas for viewing in the electronmicroscope were selected after examination by light microscopy of toluidine blue-stained semithin $(1 \mu \mathrm{m})$ sections. Ultrathin sections were double stained with uranyl acetate and lead citrate, and examined in an AEI EM801 electronmicroscope. Blocks from the choroid plexus initially fixed and stored in buffered formalin were washed, transferred to buffered glutaraldehyde, and processed for electronmicroscopy by identical methods.

\section{NECROPSY FINDINGS}

The body was that of an obese woman (weight $57 \mathrm{~kg}$, height $1580 \mathrm{~mm}$ ).

Cardiovascular system There was no macroscopic abnormality of the heart (weight $280 \mathrm{~g}$ ). Coronary atheroma was slight, and histologically there was no evidence of infarction or ischaemic fibrosis. There was slight atheroma of the abdominal aorta but there was no occlusion of major arteries, and histological sections of the carotid and vertebral arteries did not show any abnormality.

Respiratory system There was a mild terminal bronchopneumonia but no other abnormality.

Gastrointestinal system The peritoneal cavity showed acute peritonitis; the fibrinous exudate was most marked over the omentum and loops of small intestine. The cause of the peritonitis was perforation of the gall bladder which contained mixed infective stones, and was acutely and chronically inflamed. The liver $(1300 \mathrm{~g})$ histologically showed extensive focal necrosis and 
contained as a coincidental finding a $90 \mathrm{~mm}$ diameter simple fibrous-walled cyst. There was no abnormality of oesophagus, stomach, intestine, or pancreas.

Genitro-urinary system Each kidney weighed $120 \mathrm{~g}$ and both had the same appearance. The cortical surface was slightly scarred and rather pale. There was no cortical or medullary atrophy, and the pelvis was not inflamed. Histologically, small scars were present in the cortex: in these, glomeruli were hyalinised and there was associated lymphocytic infiltration. Medium sized arteries showed slight musculo-elastic hyperplasia and some afferent arterioles were hyalinised. With specialised staining there was no evidence of glomerular basement membrane thickening, wire loop formation, or fibrin deposition. The bladder was chronically inflamed.

Haemopoietic and lymphoreticular system The spleen (140 g) did not show "onion skin" fibrosis and the pulp was histologically normal. Lymph nodes and bone marrow were also histologically normal.

Endocrine system The pituitary gland did not show hyalinisation of basophil cells. Adrenal, thyroid, parathyroid glands and ovaries were normal.

Bone Sections from rib and vertebral column were examined. The bone trabeculae were normal. The bone marrow was active and contained normal cellular precursors.

Central nervous system The brain did not show any macroscopic abnormality but, histologically, in many sections from the deep white matter of the hemispheres and in the basal ganglia small blood vessels showed lymphocytic cuffing (Fig. 1) and perivascular deposition of lipochrome. Corpora amylacea were prominent around the lateral ventricles. There was no demyelination.

The spinal cord showed softening, shrinkage, and discolouration of the T4, 5, and 6 segments. Histologically an area of necrosis was identified extending from T4 to T6 segment (Fig. 2). This lesion involved the posterior columns predominantly, but also involved the medial aspect of the lateral columns (left more than right). The lateral corticospinal tracts were necrotic but the spinocerebellar tracts were preserved. The necrosis was old: numerous lipid-containing macrophages were present in the substance of the spinal cord and around small blood vessels. Occasional lymphocytes were seen but there was no acute inflammatory reaction. Many of the neurones of the posterior horn and of the nucleus dorsalis on each side had been destroyed though some cells persisted as pale homogeneous "ghosts" (Fig. 3).

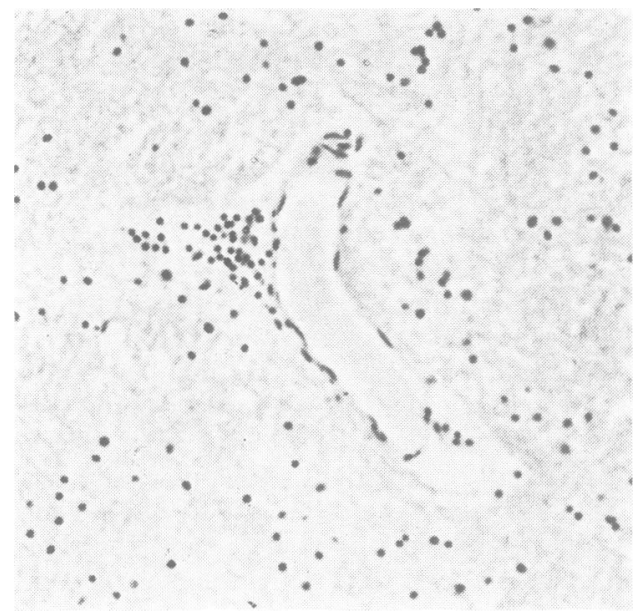

Fig. 1 Lymphocytic infiltration of perivascular space of cerebral blood vessel. $H$ and $E \times 100$.

The interomediolateral cell column was not significantly damaged. Numerous corpora amylacea were seen in sections adjacent to the necrotic segments. The main spinal arteries in sections from T4, 5, 6 segments were not thickened or obliterated but small blood vessels showed hyaline thickening and slight lymphocytic cuffing (Fig. 4). A posterior root in a section of the necrotic cord was partially necrotic (Fig. 5): this lesion was also old. The meninges in the region of the necrotic segments showed slight lymphocytic infiltration but there was no generalised meningitis. There was no abnormality of dorsal root ganglia or evidence of primary demyelination.

Light microscopy of $1 \mu \mathrm{m}$ epoxy sections from the cerebrum disclosed a generally normal appearance with the single exception that in some blocks from the cortical grey matter some small blood vessels were thickened. Similarly, in the choroid plexus the only detectable abnormality was a mild thickening of some vessels. Electronmicroscopy showed that in both cerebrum (Fig. 6) and choroid plexus (Fig. 7) this thickening was caused by an accumulation of heterogeneous dense material in the Virchow-Robin space. The bulk of this material was collagen (Fig. 6) but variable amounts of amorphous, granular, or membranous material were also present. The basement membranes appeared normal and dense "organised" deposits, fibrin, fibrillar bodies, or C type virus particles were not seen in relation to any component of the vessel walls.

Intracisternal tubuloreticular structures (TRS) were not seen in endothelial cells despite a careful search of both cerebrum and choroid. However, 


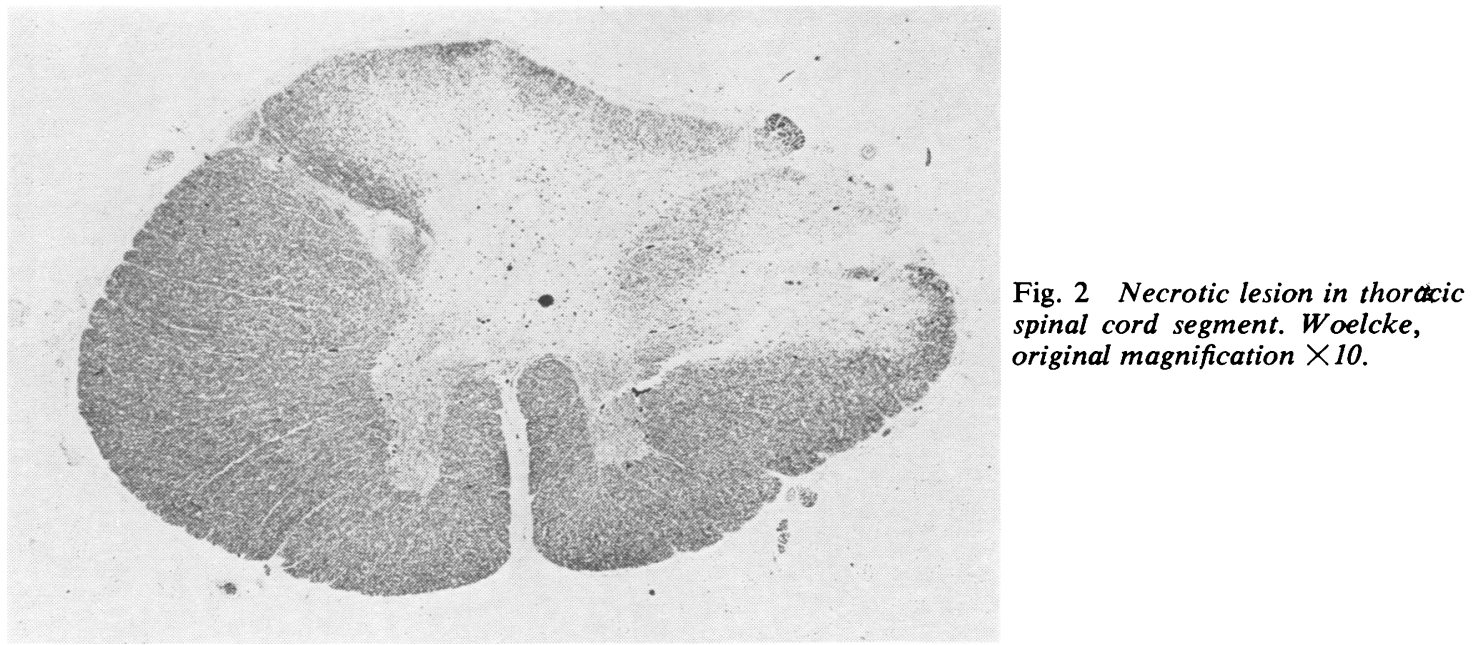

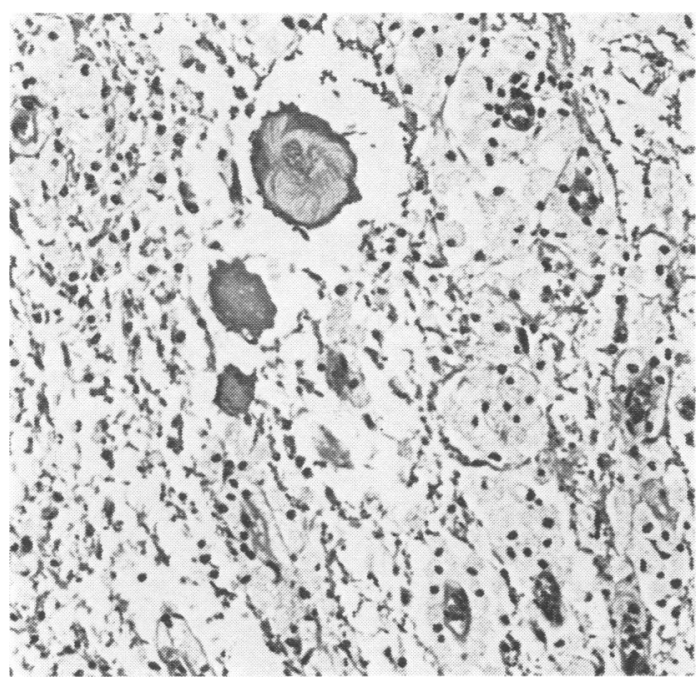

Fig. 3 Necrotic lesion in spinal cord contains lipophages and necrotic neurones. $H$ and $E \times 100$.

these cells were notable for their content of small membrane-bound, variably dense, microtubulecontaining bodies, the so-called "rod-shaped tubular bodies" of Weibel and Palade (1964) (Figs. 7b, 8). These were more numerous and prominent in the present case than in the controls which consisted of both necropsy (for example, multiple sclerosis, Huntington's chorea, and nonneurological cases) and biopsy (dementia, suspected viral encephalitis, etc.) human material. The endothelial cells appeared otherwise normal. Pathological appearances in the white matter were few, the majority of axons and sheaths being apparently normal or only altered by artefact. No clearly identifiable naked axons were seen but a few bizarre sheaths were found (Fig. 9) which differed distinctly from those seen in the control material. Such sheaths were characterised by the presence within dilatations, of ordered filamentous lattice aggregates. The dilatations also contained heterogeneous dense aggregates and some membranous bodies. Throughout the white matter occasional macrophage-like cells were seen, characterised by their abundant cytoplasm and numerous pleomorphic lysosomes.

Eyes and optic nerve The eyeballs were histologically normal but the left optic nerve was thin, and showed gliosis and nerve fibre loss (Fig. 10). Some of the small blood vessels in the nerve sheath were thickened. The right optic nerve was normal. Peripheral nerve In the sections examined there was no abnormality.

Striated muscle Sections from the upper limbs were normal but sections from the right and left quadriceps showed severe neurogenic atrophy.

\section{Discussion}

There are numerous reports of neurological symptoms in systemic lupus erythematosus and involvement of the central nervous system occurs in about $25 \%$ of cases (Fulford et al., 1972). In most cases, however, the neurological signs are set against the background of the systemic disease, and their interpretation is, therefore, relatively easy. Neurological presentation of the disease is uncommon and is usually followed by progression to the systemic form of the disease. In the present case the presentation of the disease was entirely neurological and, while the duration 


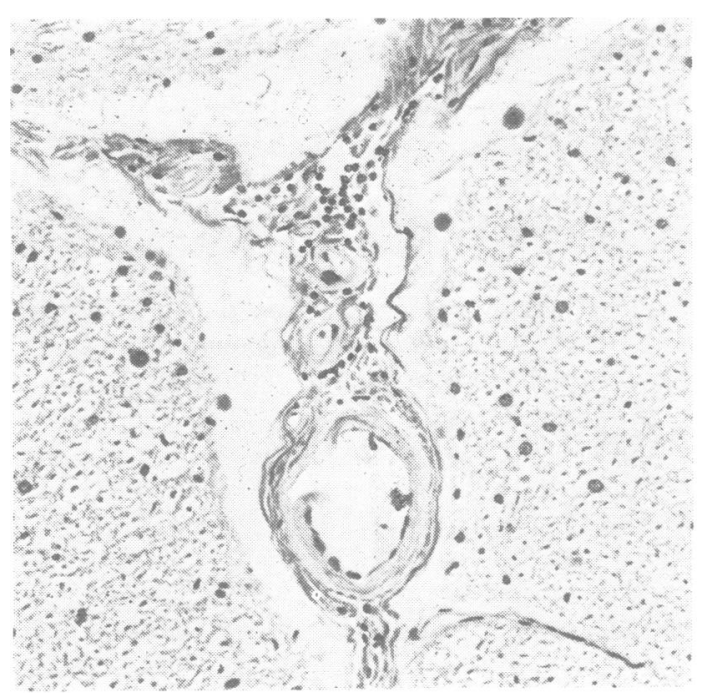

(a)

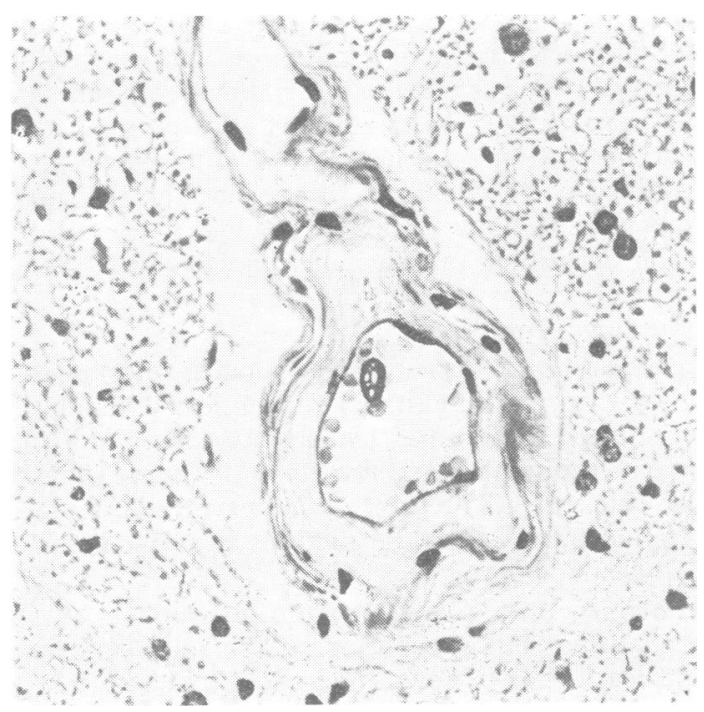

(b)

Fig. 4. Thickening and perivascular lymphocytic cuffing of spinal cord blood vessels. $H$ and $E(a) \times 100$ (b) $\times 250$.

of the disease was at least three years, there was no evidence at necropsy of involvement of systems other than the central nervous system. A further unusual feature of the case was the predominance of the spinal cord involvement. There are relatively few detailed descriptions of the pathology of the spinal cord in systemic lupus erythematosus. Vascular changes similar to those seen in the brain have been described in spinal cord blood vessels

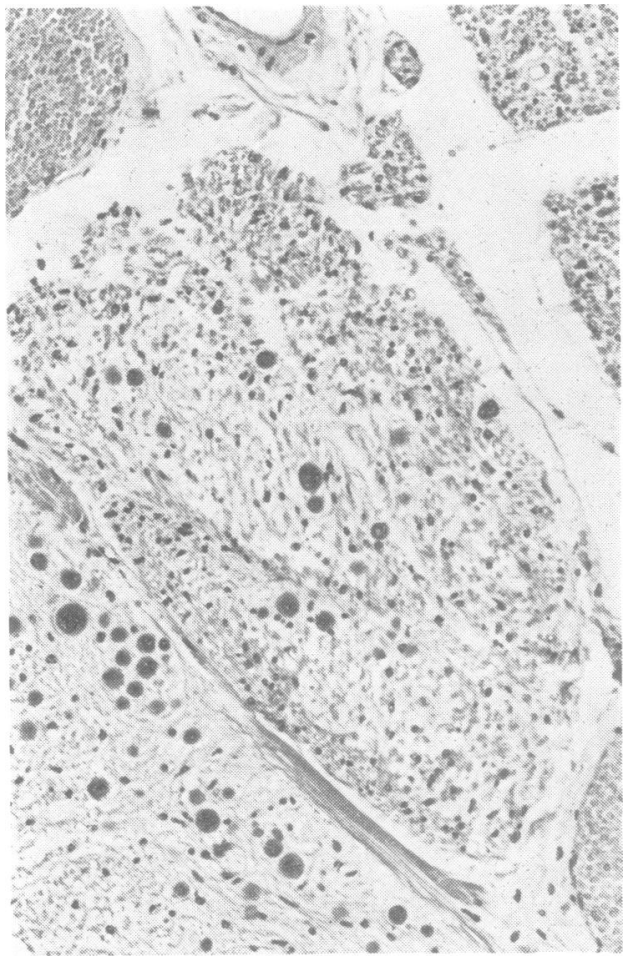

Fig. 5 Old necrotic lesion involving spinal cord and dorsal root. Note the numerous corpora amylacea. $H$ and $E$, original magnification $\times 100$.

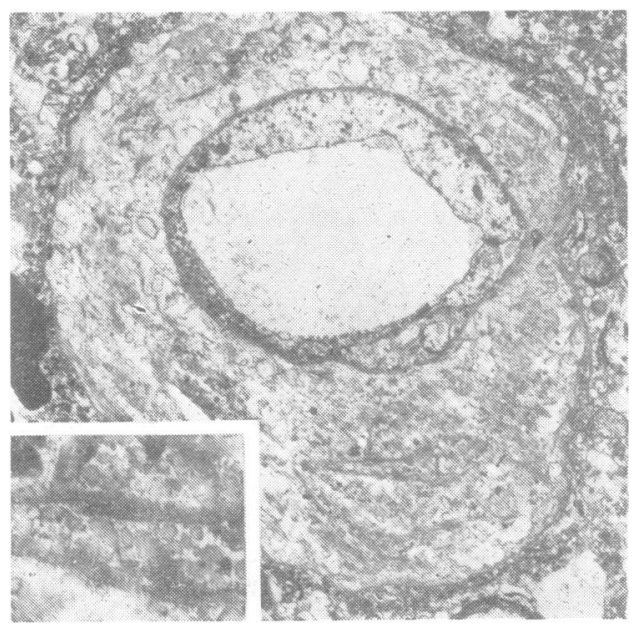

Fig. 6 Thickened Virchow Robin space in cerebral blood capillary, original magnification $\times 4250$; inset shows longitudinally and transversely sectioned collagen fibrils, original magnification $\times 16000$. 


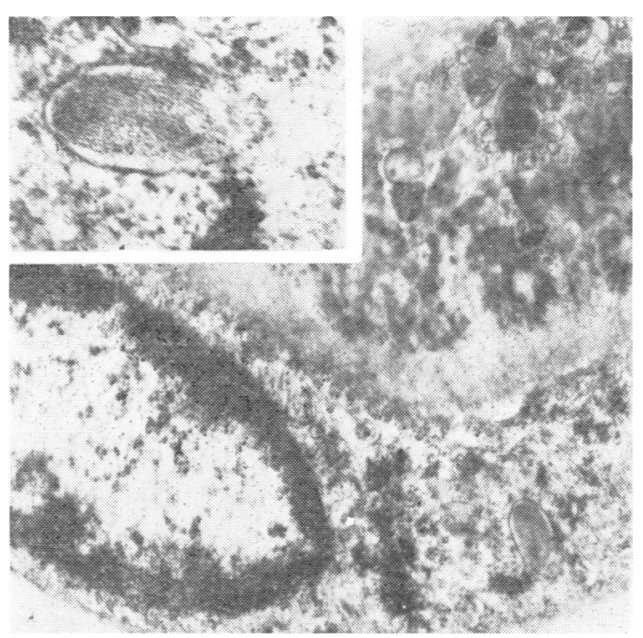

Fig. 7 Deposition of heterogeneous electron dense material below the endothelial basement membrane of an arteriole in the choroid plexus, original magnification $\times 18270$; inset higher magnification showing structure of tubular body in endothelial cell, original magnification $\times 50000$. with myelomalacia in several reports (Piper, 1953; Scheinberg, 1956; de Morsier, 1962; Penn and Rowan, 1968). Few descriptions of the spinal nerve roots are available although De Morsier (1962) describes demyelination of dorsal and ventral roots, and Bailey et al. (1956) describe necrosis of dorsal root ganglia. In the present case the persistent chest pain was thought initially to be caused by ischaemic heart disease, and it was three years after the initial presentation that a diagnosis of neuritic pain was made. The finding of necrosis in a posterior nerve root related to the necrotic thoracic segment of the spinal cord probably at least partially explains the chest pain. We have not found such a lesion previously reported in the literature on SLE.

There have been many references in the literature to similarities between multiple sclerosis and SLE, both in clinical presentation (Fulford et al., 1972) and in immunopathology (Holmes et al., 1967), and some workers have described demyelination as opposed to ischaemic necrosis (April and Vansonnenberg, 1976). In the present case the clinical presentation strongly resembled multiple sclerosis, but the pathological features of that disease were not observed. April and Vansonnenberg (1976) describe demyelination, but they ob-

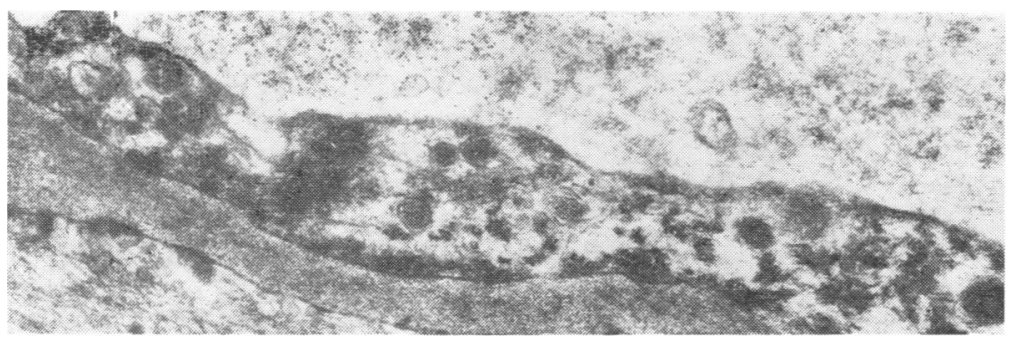

Fig. 8 Numerous dense "tubular bodies" in endothelial cell of cerebral arteriole. Note the longitudinally (large arrow) and transversely (small arrow) sectioned internal tubules. Original magnification $\times 23000$.
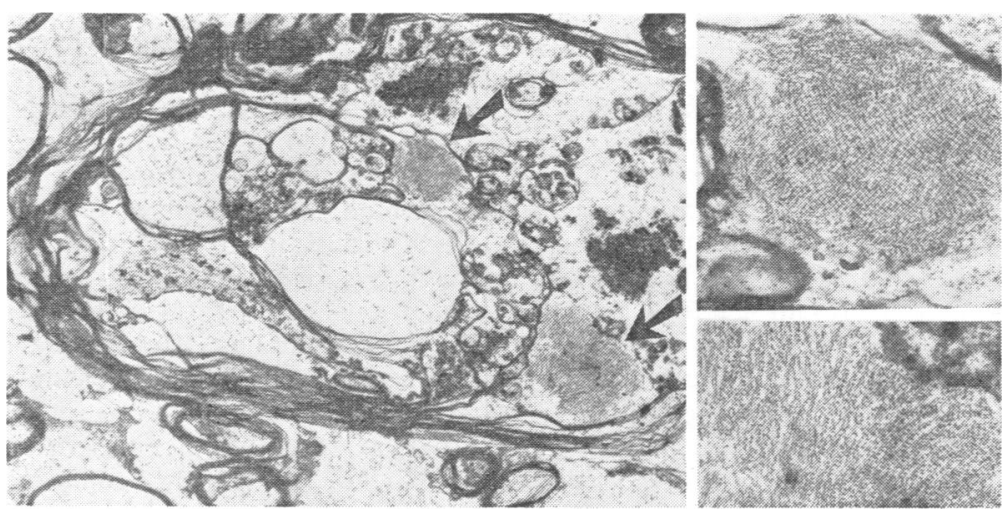

Fig. 9 Latticed fibrillar inclusions (arrows) and heterogeneous debris in degenerate cerebral myelin sheath, original magnification $\times 5500$; higher magnification of the inclusions which are cut in different planes, reveals typical Hirano body-like latticed filament substructure. Both original magnification $\times 30000$. 
(a)
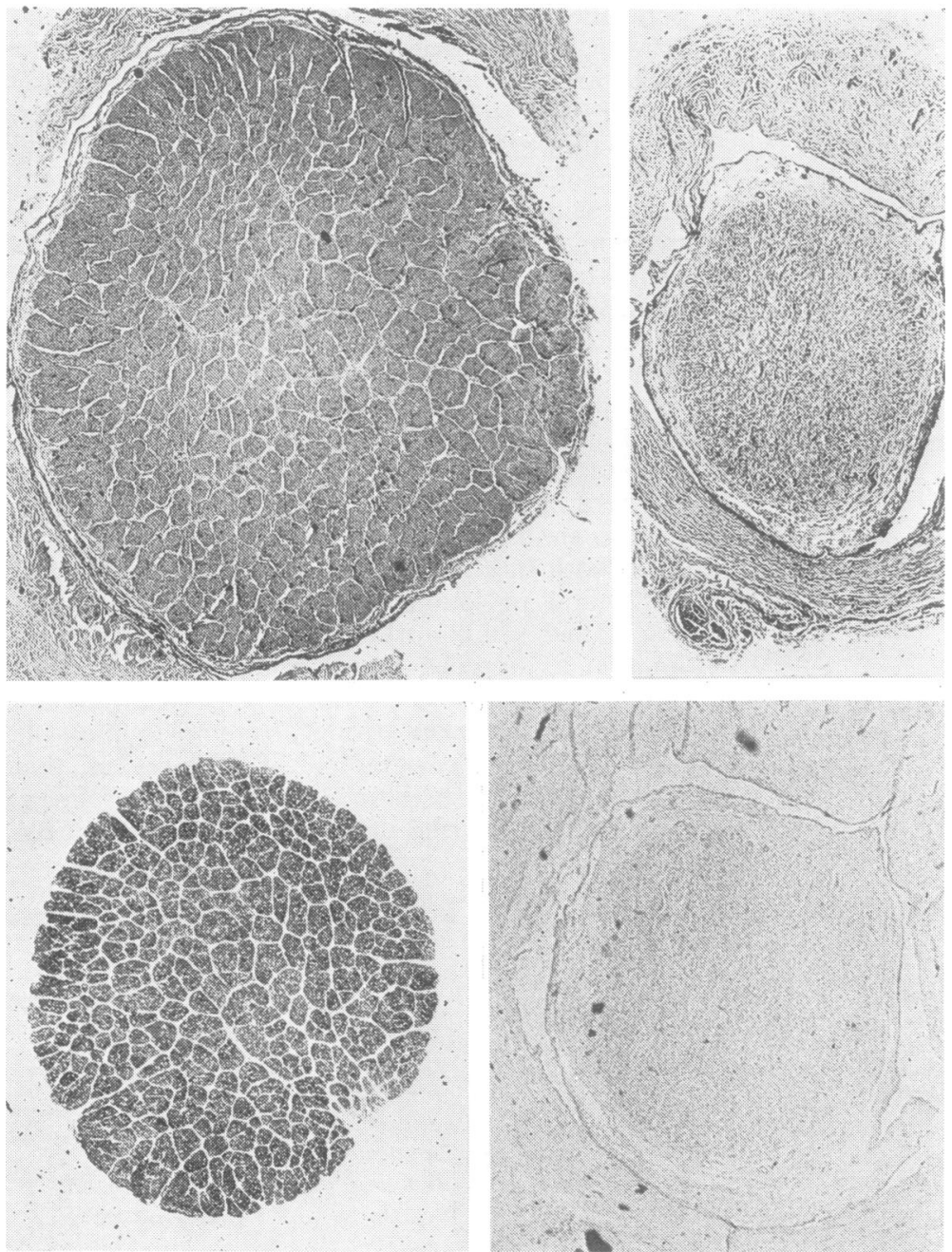

(c)

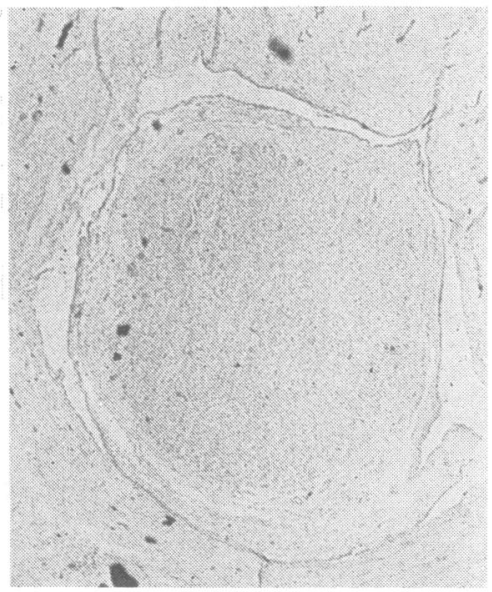

$(d)$
Fig. 10 Comparison of right $(a, c)$ and left $(b, d)$ optic nerves, the latter showing shrinkage and gliosis (a) and (b) $H$ and $E$; (c) and (d) Woelcke, original magnification in all $\times 12$. served only "a few intact neural fibres." Rarefaction in other areas which stained for myelin was attributed to considerable dilatation and vacuolation of nerve fibres. Their description, therefore, differs significantly from that of classical chronic multiple sclerosis in which nerve fibre preservation is relatively good.

Lattice fibrillar inclusions of the kind found in a few degenerating myelin sheaths in the present case have previously been seen within the oligodendroglial (Cavanagh et al., 1971) and Schwann cell (Spencer and Thomas, 1974; Hirano and Dembitzer, 1976; Hirano, 1978) adaxonal cytoplasmic compartments in pathologically altered animal tissue. However, in Hirano's studies, which were concerned with mutant Syrian hamsters with hindleg paralysis, similar though smaller inclusions were also found, though much less commonly, in control animals. In these situations their fine structure was indistinguishable from that of the classic Hirano bodies initially described in Sommer's sector of the hippocampus in Guamian amyotrophic lateral sclerosis-Parkinsonism-dementia complex (Hirano et al., 1968). Similar though often smaller inclusions have also been seen in other circumstances-for example, in cerebellar tumours (Gessaga and Anzil, 1975), in normal and kuruinfected cerebellum (Field and Raine, 1968), in 
muscle disease (Neville, 1973), and within neuronal nuclei in both normal and pathological CNS (Feldman and Peters, 1972; Cragg, 1976).

Detailed studies of Hirano bodies have shown that they are almost invariably confined to the hippocampus, and that they occur in normal human brain from the age of 10 years onwards and increasingly with age, being present in all brains over the age of 80 years (Ogata et al., 1972; Schochet and McCormick, 1972). They are found to be increased in various pathological situations, most noticeably in Alzheimer's disease (Schochet and McCormick, 1972; Gibson and Tomlinson, 1977).

The rarity of the inclusions resembling Hirano bodies in the present case and the absence of light microscopic evidence of demyelination make it seem unlikely that they indicate a significant generalised oligodendroglial defect such as was proposed by Hirano (1978) to account for the demyelination in mutant Syrian hamster. Since Hirano bodies within the sheaths of axons have not, to our knowledge, been reported previously from normal human CNS we conclude that they are abnormal, but rare, nonspecific byproducts resulting from secondary degenerative changes in the oligodendroglia. Our conclusion, both from reviewing the literature and from our observations in the present case, is that there is little firm evidence of primary demyelination in SLE. The observed myelin abnormalities can be explained as part of the ischaemic necrosis or as secondary to Wallerian degeneration.

Several other points of clinical interest emerge from this case. The beneficial effect of steroid therapy on the vision of the left eye was very striking: despite the fact that the patient had been totally blind in this eye for two years, she recovered vision in the left upper field quadrant. The explanation of this recovery is difficult, but one can only assume that oedema was a factor in the blindness. The severe gall bladder disease and peritonitis were probably aggravated by the steroid therapy, while the paucity of abdominal clinical signs at the time of death (particularly rigidity) was presumably the result of the spinal cord lesion.

Electron microscopy of the apparently minimally affected cerebrum in this case is in keeping with other studies of SLE which have pointed to the primarily vascular nature of the lesions in this disease. Failure to find the frequently reported (Norton, 1969; Bariety et al., 1973; Helder and Feltkamp-Vroom, 1976) but nonspecific tubuloreticular structures in the endoplasmic reticulum or nuclear envelope of endo- thelial cells in the cerebrum and choroid plexus was not unexpected considering the relatively mild involvement of these tissues in this case. Norton (1969), for example, has pointed out that while they may be found frequently in affected tissue they may be infrequent or absent in uninvolved tissue from the same individual. The finding in the present case of large numbers of rod-shaped tubular bodies in endothelial cells, particularly in the cerebrum, has not previously been reported in SLE but may be of significance in several respects. These are normally occurring but poorly understood specific organelles of endothelial cells (Ghadially, 1975), originally described by Weibel and Palade (1964). They have since been shown to arise directly from the cisterns of the Golgi apparatus (Sengel and Stoebner, 1970), and there is evidence that they may discharge their contents into the blood plasma after fusion with the endothelial plasma membrane (Ohsugi and Hirano, 1977). Their function is not yet fully elucidated, but they may secrete factors which enhance blood coagulation (Burri and Weibel, 1968), or affect blood pressure (Bertini and Santolaya, 1970). While there is disagreement as to how common they are in normal adult human CNS (Herlinger et al., 1974; Hirano, 1976), it is clear that in certain conditions, most notably in tumours (Kawamura et al., 1974), they are present in increased numbers, size, and variety of form. Dense compact forms of tubular bodies are only rarely seen in involved tissue in SLE. For example, Norton (1970) found numerous tubular reticular structures but no tubular bodies in vessels from muscle biopsy samples in cases of SLE and dermatomyositis. However, in scleroderma the converse was true, and Norton attributed this to differences in the form of the microvascular injury in these conditions. He suggested that, whereas in SLE and dermatomyositis this injury was acute and episodic, in scleroderma it was more chronic and sustained.

The finding of the tubular bodies in the present case raises the question of a possible relationship between variants of such bodies and the tubuloreticular structures reported commonly in SLE. Thus, while the tubular bodies found in our case were mainly of the dense, compact variety not likely to be confused with tubuloreticular inclusions, there is a striking similarity between many of the published micrographs illustrating the latter (Norton, 1969; Hass and Yunis, 1970; Bariety et al., 1973; Helder and Feltkamp-Vroom, 1976), and those illustrating the vacuolar forms of tubular bodies (Kawamura et al., 1974; Ohsugi and Hirano, 1977). This apparent overlap calls into question the validity of much of the published 
data on the incidence of tubuloreticular structures in SLE for, despite claims to the contrary (Kawamura et al., 1974), the two types of inclusion cannot reliably be distinguished in pathological material by objective morphological criteria.

\section{References}

April, R. S., and Vansonnenberg, E. (1976). A case of neuromyelitis optica (Devic's syndrome) in systemic lupus erythematosus. Neurology (Minneapolis), 26, 1066-1070.

Bailey, A. A., Sayre, G. P., and Clark, E. C. (1956). Neuritis associated with systemic lupus erythematosus. Archives of Neurology and Psychiatry (Chicago), 75, 251-259.

Bariety, J., Richer, D., Appay, M. D., Grossetete, J., and Callard, P. (1973). Frequency of intraendothelial "virus-like" particles: an electron microscopic study of 376 human renal biopsies. Journal of Clinical Pathology, 26, 21-24.

Bertini, F., and Santolaya, R. (1970). A novel type of granules observed in toad endothelial cells and their relationship with blood pressure active factors. Experientia, 26, 522-523.

Burri, P. H., and Weibel, E. R. (1968). Beeinflussing einer spezifischen cytoplasmatischen organelle von endothelzellen durch adrenalin. Zeitschrift für Zellforschung und Mikroskopische Anatomie, 88, 426440.

Cavanagh, J. B., Blakemore, W. F., and Kyu, M. H. (1971). Fibrillary accumulations in oligodendroglial processes of rats subjected to portocaval anastomosis. Journal of the Neurological Sciences, 14, 143-152.

Cragg, B. G. (1976). Ultrastructural features of human cerebral cortex. Journal of Anatomy, 121, 331-362.

De Morsier, G. (1962). Lupus erythemateux disséminé avec lesions encephalomedullaires et troubles mentaux. World Neurology, 3, 629-658.

Feldman, M. L., and Peters, A. (1972). Intranuclear rods and sheets in rat cochlear nucleus. Journal of Neurocytology, 1, 109-127.

Feng, P. H., Cheah, P. S., and Lee, Y. K. (1973). Mortality in systemic lupus erythematosus: a 10year review. British Medical Journal, 4, 772-774.

Field, E. J., and Raine, C. S. (1968). Lattice fibrillar and spiral structures in normal chimpanzee and human brain. The Veterinary Record, 5, 495-497.

Fulford, K. W. M., Catterall, R. D., Delhanty, J. J., Doniach, D., and Kremer, M. (1972). A collagen disorder of the nervous system presenting as multiple sclerosis. Brain, 95, 373-386.

Gessaga, E. C., and Anzil, A. P. (1975). Rod-shaped filamentous inclusions and other ultrastructural features in a cerebellar astrocytoma. Acta Neuropathologica, 33, 119-127.

Ghadially, F. N. (1975). Ultrastructural Pathology of the Cell. Butterworths: London.

Gibson, P. H., and Tomlinson, B. E. (1977). Numbers of Hirano bodies in the hippocampus of normal and demented people with Alzheimer's disease. Journal of the Neurological Sciences, 33, 199-206.

Haas, J. E., and Yunis, E. J. (1970). Tubular inclusions of systemic lupus erythematosus. Ultrastructural observations regarding their possible viral nature. Experimental and Molecular Pathology, 12, 257263.

Helder, A. W., and Feltkamp-Vroom, T. M. (1976). Tubulo-reticular structures and antinuclear antibodies in autoimmune and non-autoimmune diseases. Journal of Pathology, 119, 49-56.

Herlinger, H., Anzil, A. P., Blinzinger, K., and Kronski, D. (1974). Endothelial microtubular bodies in human brain capillaries and venules. Journal of Anatomy, 11, 205-209.

Hirano, A. (1976). Further observations of the fine structure of pathological reaction in cerebral blood vessels. In The Cerebral Vessel Wall. Edited by J. Cervós-Navarro, E. Betz, F. Matakas, and R. Wullenweber. Raven Press: New York.

Hirano, A. (1978). A possible mechanism of demyelination in the Syrian hamster with hindleg paralysis. Laboratory Investigation, 38, 115-121.

Hirano, A., and Dembitzer, H. M. (1976). Eosinophilic rod-like structures in myelinated fibres of hamster spinal roots. Neuropathology and Applied Neurobiology, 2, 225-232.

Hirano, A., Dembitzer, H. M., Kurland, L. T., and Zimmerman, H. M. (1968). The fine structure of some intraganglionic alterations. Journal of Neuropathology and Experimental Neurology, 27, 167182.

Holmes, F. F., Stubbs, D. W., and Larsen, W. E. (1967). Systemic lupus erythematosus and multiple sclerosis in identical twins. Archives of Internal Medicine, 119, 302-304.

Kawamura, J., Kamijyo, Y., Sunaga, T., and Nelson, E. (1974). Tubular bodies in vascular endothelium of a cerebellar neoplasm. Laboratory Investigation, 30, 358-365.

Neville, H. E. (1973). Ultrastructural changes in muscle disease. In Muscle Biopsy: A Modern Approach, pp. 383-470. Edited by V. Dubowitz and M. H. Brooke. W. B. Saunders: London.

Norton, W. L. (1969). Endothelial inclusions in active lesions of systemic lupus erythematosus. Journal of Laboratory and Clinical Medicine, 74, 369-379.

Norton, W. L. (1970). Comparison of the microangiopathy of systemic lupus erythematosus, dermatomyositis, scleroderma and diabetes mellitus. Laboratory Investigation, 22, 301-308.

Ogata, J., Budzilovich, G. N., and Cravioto, H. (1972). A study of rod-like structures (Hirano bodies) in 240 normal and pathological brains. Acta Neuropathologica, 21, 61-67.

Ohsugi, T., and Hirano, A. (1977). Tubular bodies in endothelial cells in meningiomas. Neuropathology and Applied Neurobiology, 3, 1-8.

Osler, W. (1900). The visceral lesions of the erythema group. British Journal of Dermatology, 12, 227245. 
Penn, A. S., and Rowan, A. J. (1968). Myelopathy in systemic lupus erythematosus. Archives of Neurology (Chicago), 18, 337-349.

Piper, P. G. (1953). Disseminated lupus erythematosus with involvement of the spinal cord. Journal of the American Medical Association, 153, 215-217.

Scheinberg, L. (1956). Polyneuritis in systemic lupus erythematosus. New England Journal of Medicine, 255, 416-421.

Schochet, S. S., and McCormick, W. F. (1972). Ultrastructure of Hirano bodies. Acta Neuropathologica, 21, $50-60$.
Sengel, A., and Stoebner, P. (1970). Golgi origin of tubular inclusions in endothelial cells. Journal of Cell Biology, 44, 223-226.

Spencer, P. S., and Thomas, P. K. (1974). Ultrastructural studies of the dying-back process II. The sequestration and removal by Schwann cells and oligodendrocytes of organelles from normal and diseased axons. Journal of Neurocytology, 3, 763783.

Weibel, E. R., and Palade, G. E. (1964). New cytoplasmic components in arterial endothelia. Journal of Cell Biology, 23, 101-112. 